\title{
ESTIMATION OF BLOOD LOSS AND FACTORS INFLUENCING BLOOD LOSS IN TRANSURETHRAL RESECTION OF PROSTATE: A PROSPECTIVE STUDY
}

\author{
Vetrichandar $S^{1}$, Aysha Shaheen $B^{2}$ \\ ${ }^{1}$ Consultant Urologist, Aysha Urology and Gynaecology Clinic, Tamilnadu Dr. MGR Medical University, Chennai. \\ ${ }^{2}$ Consultant Urogynecologist, Aysha Urology and Gynaecology Clinic, Tamilnadu Dr. MGR Medical University, Chennai.
}

\section{ABSTRACT}

\section{BACKGROUND}

Transurethral resection of the prostate remains the gold standard treatment of BPH. Though, the mortality of TURP has decreased to less than 1\%, the significant morbidity of the bleeding complications still persists. Hence, blood losses must be replaced promptly to ensure an optimal blood volume and oxygen transport. The aim of this study is to study the factors influencing blood loss during TURP.

\section{MATERIALS AND METHODS}

This is a prospective study on patients with BPH treated with transurethral resection of prostate at Kilpauk Medical College and Government Royapettah Hospital between January 2012 and February 2013. Patient who require surgery because of severe symptoms or retention of urine secondary to BPH were included in this study. Total number of patients included in the study is 40 . Informed consent was obtained from all patients.

\section{RESULTS}

Out of multiple variables analysed in 40 patients, it is concluded from our study that the extent of blood loss associated with TURP is multifactorial and it is impossible to measure the effects of single factor (e.g. local vascularity) while controlling all others. The ' $\mathrm{P}$ ' value was significant for the weight of resected tissue and longer operative time. Hence, a meticulously performed TURP with reasonable speed and attention to details is the way forward in reducing perioperative blood loss.

\section{CONCLUSION}

The extent of blood loss associated with TURP is multifactorial and it is impossible to measure the effects of single factor (e.g. local vascularity) while controlling all others. Of the measured factors, the weight of resected tissue and longer operative time are clearly most important factors which significantly increase the blood loss. Hence, a meticulously performed TURP with reasonable speed and attention to details is the way forward in reducing perioperative blood loss.

\section{KEYWORDS}

BPH, TURP, Blood Loss, Complications of TURP.

HOW TO CITE THIS ARTICLE: Vetrichandar S, Shaheen AB. Estimation of blood loss and factors influencing blood loss in transurethral resection of prostate: a prospective study. J. Evolution Med. Dent. Sci. 2016;5(103):7592-7598, D0I: 10.14260/jemds/2016/1717

\section{BACKGROUND}

The increase in life expectancy of the population has led to an increase in the prevalence of geriatric problems like benign prostatic hyperplasia. Benign Prostatic Hyperplasia (BPH) has become a significant public health problem adding to the cost of healthcare in the society. BPH occurs usually in the peripheral zone.1,2

Though, many treatment modalities are available, ${ }^{3}$ transurethral resection of the prostate remains the gold standard treatment of BPH and the mortality of TURP has decreased to less than $1 \%$. The significant morbidity of the bleeding complications still persists because of its peculiar vascular anatomy. ${ }^{4}$ An ideal complication free treatment of BPH still remains a mirage. From treatment by medicine using alpha blockers and 5 alpha reductase inhibitors to laser

Financial or Other, Competing Interest: None.

Submission 22-07-2016, Peer Review 17-08-2016,

Acceptance 23-08-2016, Published 26-12-2016.

Corresponding Author:

Dr. Vetrichandar $S$,

No 83, Thirumangalam Road,

Villiwalkam,

Chennai-49.

E-mail:drsvchandar@gmail.com

DOI: $10.14260 /$ jemds/2016/1717 prostatectomies, all treatment modalities have their own side effects.

Indicated patients are subjected to TURP and blood loss must be replaced promptly to ensure an optimal blood volume and oxygen transport.

Various studies have been done to determine the factors that influence bleeding during the procedure and estimation of blood loss during TURP will help to assess the need for replacement, thus reducing morbidity and mortality. We have studied those factors in 40 patients.

\section{Inclusion Criteria}

1. All patients undergoing TURP.

\section{Exclusion Criteria}

1. Patients on anticoagulants and bleeding disorders.

2. Patients with renal failure.

3. Patients treated early by 5 -alpha reductase inhibitor.

Patient's assessment began from outpatient department with detailed history including type of presentation, acute retention or not and physical examination including digital rectal examination. 


\section{Laboratory Examination Include}

1. Complete blood picture, bleeding time, clotting time.

2. Preoperative haemoglobin.

3. Blood sugar, urea, serum creatinine, electrolytes.

4. Urine analysis and culture and sensitivity were performed.

5. Chest x-ray and ECG.

6. Ultrasound of kidney, bladder and prostate.

7. Uroflowmetry in selected patients.

The prostate was imaged in the coronal and sagittal sections and the volume calculated using ellipsoid formula.

(Volume = height $\mathrm{x}$ width $\mathrm{x}$ length $\mathrm{x}$ 0.52)

All TURP in this study were conducted under spinal anaesthesia given by experienced anaesthetists.

The operation was carried out as patients in the lithotomy position with a Karl Storz 24-fr resectoscope with sterile water as an irrigant.

Nesbit's and Blandy's technique ${ }^{5}$ were used for performing TURP.

After proper positioning and draping, cystourethroscopy was done by $22-\mathrm{Fr}$ sheath with $30^{\circ}$ telescope to assess Urethra, Veru, Prostate, Ureteric orifice and Bladder.

Urethral dilatations were done only in cases where passage of scope was not easy.

A 24-Fr continuous irrigation resectoscope with passive cutting working element was used for resection. Monopolar diathermy on pure mode was used.

Haemostasis was done by pin point coagulation and roller ball coagulation.

The resection time was measured from the insertion to the removal of resectoscope. The volume of irrigation fluid was measured.
The sample were collected from the irrigation fluid bucket; after continuous stirring for 5 minutes samples were taken and sent for haemoglobin estimation. Haemoglobin measurement was done by a photometer Sysmex KX-21.

All resected prostatic tissues were dried, weighed and dispatched for histological examination.

\section{The Blood loss was Estimated using the Formula:}

\begin{tabular}{|c|c|}
\hline & Hb content of the irrigant (gm. /L) X Volume (L) x1000 \\
\hline blood loss in $\mathrm{ml}=$ & Blood Hemoglobin $(\mathrm{gm} / \mathrm{dl}) \times 5.2$ \\
\hline
\end{tabular}

The result will be assessed using Student's 'T' test, Pearson product, Moment correlation coefficient and Chisquare test as appropriate.

\section{RESULTS}

DSA Descriptive.

\begin{tabular}{|c|c|c|c|c|c|}
\hline & N & Mini. & Max. & Mean & $\begin{array}{c}\text { Std. } \\
\text { Deviation }\end{array}$ \\
\hline Age & 40 & 56 & 85 & 68.93 & 7.467 \\
\hline Preop Hb & 40 & 9.4 & 13.6 & 11.293 & 1.0131 \\
\hline Gland Size & 40 & 30 & 82 & 53.80 & 13.960 \\
\hline $\begin{array}{c}\text { Operating } \\
\text { Time }\end{array}$ & 40 & 35 & 70 & 52.38 & 10.252 \\
\hline $\begin{array}{c}\text { Resected } \\
\text { Tissue Wt. }\end{array}$ & 40 & 16 & 39 & 26.30 & 6.696 \\
\hline $\begin{array}{c}\text { Calc. Blood } \\
\text { Loss }\end{array}$ & 40 & 84.6 & 240.5 & 129.790 & 37.1299 \\
\hline $\begin{array}{c}\text { Valid N } \\
\text { (List Wise) }\end{array}$ & 40 & & & \\
\hline \multicolumn{7}{|c|}{ Table 1. Descriptive Statistics } \\
\hline
\end{tabular}

\begin{tabular}{|c|c|c|c|c|c|c|c|}
\hline & & Age & $\begin{array}{c}\text { Preop } \\
\text { Hb }\end{array}$ & $\begin{array}{l}\text { Gland } \\
\text { Size }\end{array}$ & $\begin{array}{l}\text { Operating } \\
\text { Time }\end{array}$ & $\begin{array}{c}\text { Resected Tissue } \\
\text { Wt. }\end{array}$ & $\begin{array}{l}\text { Calc. Blood } \\
\text { Loss }\end{array}$ \\
\hline \multirow[t]{3}{*}{ Age } & Pearson Correlation & 1 & -.269 & -.184 & .153 & -.227 & -.214 \\
\hline & Sig. (2-tailed) & . & .093 & .255 & .346 & .159 & .185 \\
\hline & $\mathrm{N}$ & 40 & 40 & 40 & 40 & 40 & 40 \\
\hline \multirow[t]{3}{*}{ Preop Hb } & Pearson Correlation & -.269 & 1 & -.025 & -.248 & .105 & .015 \\
\hline & Sig. (2-tailed) & .093 & . & .877 & .124 & .520 & .925 \\
\hline & $\mathrm{N}$ & 40 & 40 & 40 & 40 & 40 & 40 \\
\hline \multirow[t]{3}{*}{ Gland Size } & Pearson Correlation & -.184 & -.025 & 1 & $.621\left(^{* *}\right)$ & $.753\left(^{* *}\right)$ & $.674(* *)$ \\
\hline & Sig. (2-tailed) & .255 & .877 & . & .000 & .000 & .000 \\
\hline & $\mathrm{N}$ & 40 & 40 & 40 & 40 & 40 & 40 \\
\hline \multirow[t]{3}{*}{$\begin{array}{c}\text { Operating } \\
\text { Time }\end{array}$} & Pearson Correlation & .153 & -.248 & $.621\left(^{* *}\right)$ & 1 & $.486\left(^{* *}\right)$ & $\left..419^{* *}\right)$ \\
\hline & Sig. (2-tailed) & .346 & .124 & .000 & . & .001 & .007 \\
\hline & $\mathrm{N}$ & 40 & 40 & 40 & 40 & 40 & 40 \\
\hline \multirow[t]{3}{*}{$\begin{array}{l}\text { Resected } \\
\text { Tissue Wt. }\end{array}$} & Pearson Correlation & -.227 & .105 & $.753\left(^{* *}\right)$ & $.486\left(^{* *}\right)$ & 1 & $.936\left(^{* *}\right)$ \\
\hline & Sig. (2-tailed) & .159 & .520 & .000 & .001 & . & .000 \\
\hline & $\mathrm{N}$ & 40 & 40 & 40 & 40 & 40 & 40 \\
\hline \multirow[t]{3}{*}{$\begin{array}{l}\text { Calc. Blood } \\
\text { Loss } \\
\end{array}$} & Pearson Correlation & -.214 & .015 & $.674(* *)$ & $.419\left(^{* *}\right)$ & $.936\left(^{* *}\right)$ & 1 \\
\hline & Sig. (2-tailed) & .185 & .925 & .000 & .007 & .000 & . \\
\hline & $\mathrm{N}$ & 40 & 40 & 40 & 40 & 40 & 40 \\
\hline
\end{tabular}

** Correlation is significant at the 0.01 level (2-tailed). 
Analysis

Correlation between Age and Calculated Blood Loss: (Table 3, Graph 1)

Minimum blood loss : $84.6 \mathrm{~mL}$.

Maximum blood loss : $240.5 \mathrm{~mL}$.

Average blood loss : $129.8 \mathrm{~mL}$.

Age of the Patients

In our study

Minimum age : 56 yrs.

Maximum age : $\quad 85$ yrs.

Average : 68.93 yrs

Correlation between Age and Calculated Blood Loss

$P$ value is 0.185 .

No significance is defined.

Correlations between Preoperative Haemoglobin and

Calculated Blood Loss: (Table 4, Graph 2)

Preoperative Haemoglobin

Preoperative $\mathrm{Hb}$ gm\%:

Minimum : $9.4 \mathrm{gm} \%$.

Maximum : $13.6 \mathrm{gm} \%$.

Average $\quad: \quad 11.29 \mathrm{gm} \%$.

$P$ value is 0.925 .

Preoperative $\mathrm{Hb}$ level is not significant.

Correlation between Gland Size and Calculated Blood Loss: (Table 5, Graph 3)

Minimum : 30 gms.

Maximum : 82 gms.

Average : 53.80 gms.

$P$ value is 0.000 .

Correlation between gland size and calculated blood loss was significant.

If the gland size is bigger, the blood loss will be more.

Correlation between Operating Time and Calculated Blood Loss: (Table 6, Graph 4)

Minimum : 35 mins.

Maximum : 70 mins.

Average : 52.38 .

$P$ value is 0.007 .

Operating time correlation with blood loss will be significant.

If resection time is longer, blood loss will be more.

Correlation between Resected Tissue Weight and Calculated Blood Loss: (Table 7, Graph 5)

Minimum : $16 \mathrm{gm}$.

Maximum : $39 \mathrm{gm}$.

Average : 26.30 .

$P$ value is 0.000 .

Correlation between Resected tissue weight and blood loss is significant.

If there is large resected tissue, the blood loss will be more.

\begin{tabular}{|c|c|c|c|}
\hline \multirow{4}{*}{ Age } & Age & $\begin{array}{c}\text { Calc. } \\
\text { Blood Loss }\end{array}$ \\
\hline \multirow{4}{*}{$\begin{array}{c}\text { Calc. Blood } \\
\text { Loss }\end{array}$} & $\begin{array}{c}\text { Pearson } \\
\text { Correlation }\end{array}$ & 1 & -.214 \\
\cline { 2 - 4 } & Sig. (2-tailed) &. & .185 \\
\cline { 2 - 4 } & $\mathrm{N}$ & 40 & 40 \\
\cline { 2 - 4 } & $\begin{array}{c}\text { Pearson } \\
\text { Correlation }\end{array}$ & -.214 & 1 \\
\cline { 2 - 4 } & Sig. (2-tailed) & .185 &. \\
\hline \multicolumn{2}{|c|}{ N } & 40 & 40 \\
\hline \multicolumn{2}{|c|}{ Table 3. Correlations between Age and } \\
Calculated Blood Loss
\end{tabular}

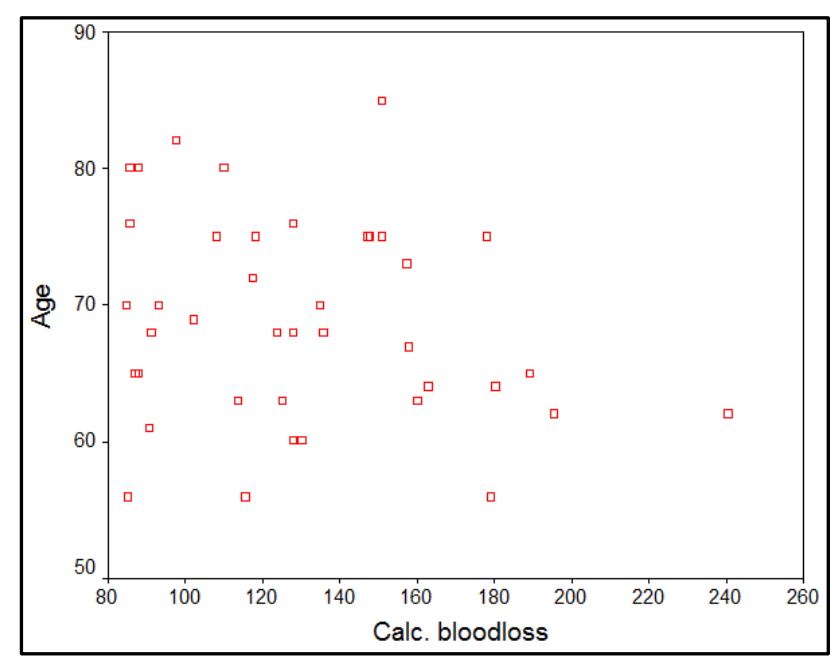

Graph 1

\begin{tabular}{|c|c|c|c|}
\hline & & $\begin{array}{c}\text { Pre-Op } \\
\text { Hb }\end{array}$ & $\begin{array}{l}\text { Calc. Blood } \\
\text { Loss }\end{array}$ \\
\hline \multirow{3}{*}{ Pre-Op Hb } & $\begin{array}{c}\text { Pearson } \\
\text { Correlation }\end{array}$ & 1 & .015 \\
\hline & Sig. (2-tailed) & . & .925 \\
\hline & $\mathrm{N}$ & 40 & 40 \\
\hline \multirow{3}{*}{$\begin{array}{l}\text { Calc. Blood } \\
\text { Loss }\end{array}$} & $\begin{array}{c}\text { Pearson } \\
\text { Correlation }\end{array}$ & .015 & 1 \\
\hline & Sig. (2-tailed) & .925 & . \\
\hline & $\mathrm{N}$ & 40 & 40 \\
\hline
\end{tabular}

Table 4. Correlations between Preoperative Haemoglobin and Calculated Blood Loss

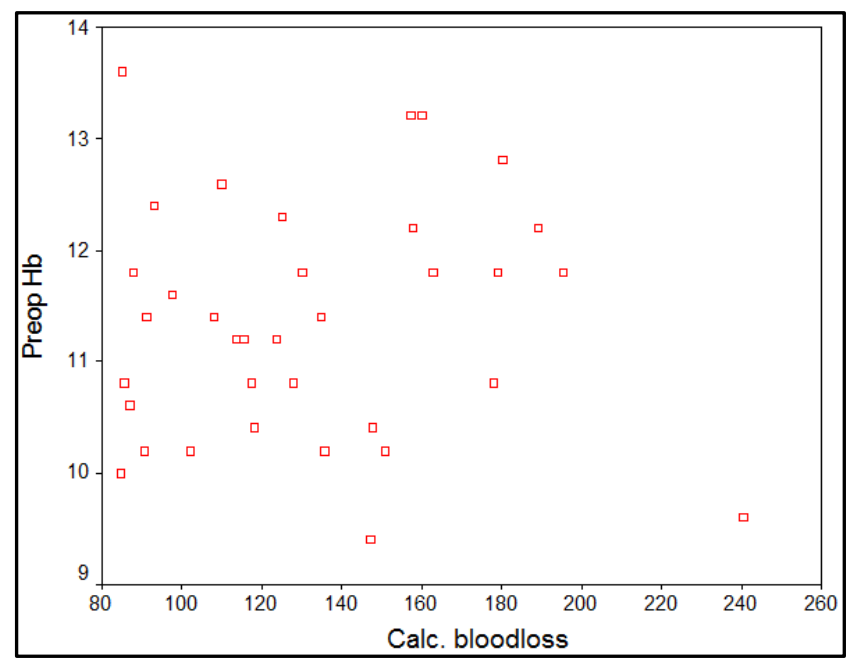

Graph 2 


\begin{tabular}{|c|c|c|c|}
\hline & & $\begin{array}{c}\text { Gland } \\
\text { Size }\end{array}$ & $\begin{array}{c}\text { Calc. } \\
\text { Blood Loss }\end{array}$ \\
\hline \multirow{3}{*}{ Gland Size } & $\begin{array}{l}\text { Pearson } \\
\text { Correlation }\end{array}$ & 1 & $.674(* *)$ \\
\hline & Sig. (2-tailed) & . & .000 \\
\hline & $\mathrm{N}$ & 40 & 40 \\
\hline \multirow{3}{*}{$\begin{array}{l}\text { Calc. Blood } \\
\text { Loss }\end{array}$} & $\begin{array}{c}\text { Pearson } \\
\text { Correlation }\end{array}$ & $.674(* *)$ & 1 \\
\hline & Sig. (2-tailed) & .000 & . \\
\hline & $\mathrm{N}$ & 40 & 40 \\
\hline & $\begin{array}{l}\text { Correlation } \\
\text { and Calculat }\end{array}$ & $\begin{array}{l}\text { tween } C \\
\text { Blood Lo }\end{array}$ & d Size \\
\hline
\end{tabular}

${ }^{* *}$ Correlation is significant at the 0.01 level (2-tailed).

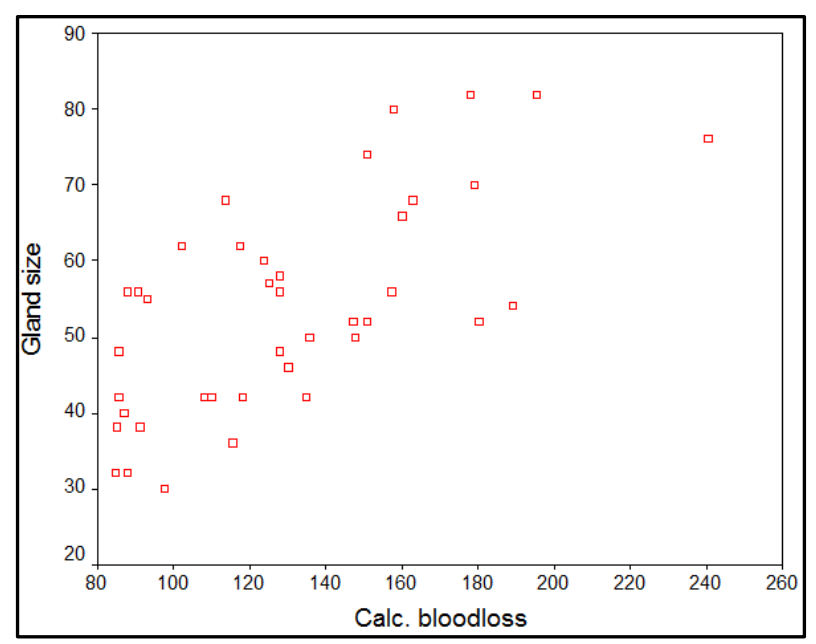

Graph 3

\begin{tabular}{|c|c|c|c|}
\hline & & $\begin{array}{l}\text { Operating } \\
\text { Time }\end{array}$ & $\begin{array}{l}\text { Calc. } \\
\text { Blood } \\
\text { Loss }\end{array}$ \\
\hline \multirow{3}{*}{$\begin{array}{l}\text { Operating } \\
\text { Time }\end{array}$} & $\begin{array}{c}\text { Pearson } \\
\text { Correlation }\end{array}$ & 1 & $.419\left(^{* *}\right)$ \\
\hline & Sig. (2-tailed) & . & .007 \\
\hline & $\mathrm{N}$ & 40 & 40 \\
\hline \multirow{3}{*}{$\begin{array}{l}\text { Calc. } \\
\text { Blood } \\
\text { Loss }\end{array}$} & $\begin{array}{c}\text { Pearson } \\
\text { Correlation }\end{array}$ & $.419\left(^{* *}\right)$ & 1 \\
\hline & Sig. (2-tailed) & .007 & . \\
\hline & $\mathrm{N}$ & 40 & 40 \\
\hline
\end{tabular}

${ }^{* *}$ Correlation is significant at the 0.01 level (2-tailed).

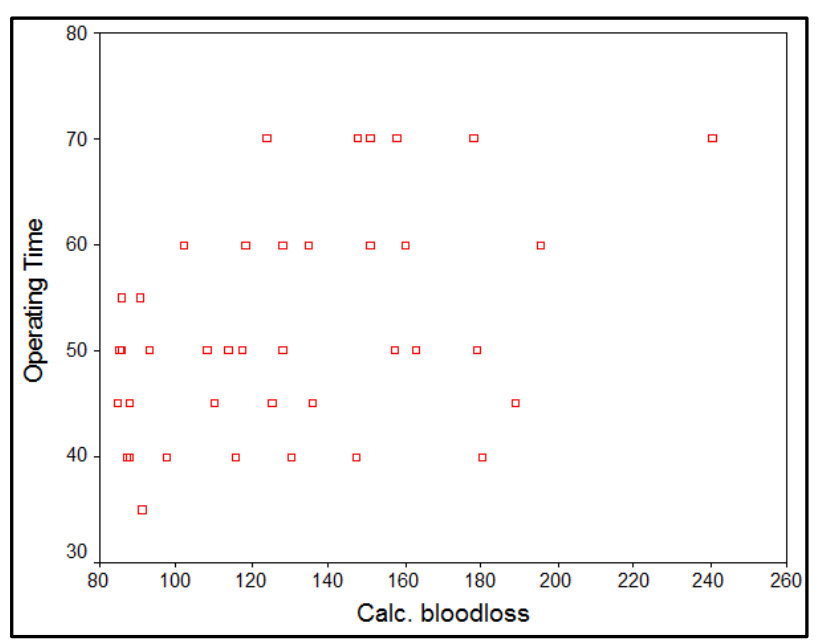

Graph 4

\begin{tabular}{|c|c|c|}
\hline & & Calc. Blood Loss \\
\hline \multirow{2}{*}{$\begin{array}{c}\text { Resected Tissue } \\
\text { Wt }\end{array}$} & Pearson Correlation & $.936(* *)$ \\
\cline { 2 - 3 } & Sig. (2-tailed) & .000 \\
\cline { 2 - 3 } & $\mathrm{N}$ & 40 \\
\hline \multicolumn{2}{|c|}{ Table 7. Correlations between Resected Tissue } \\
Weight and Calculated Blood Loss \\
\hline \multirow{2}{|c|}{}
\end{tabular}

${ }^{* *}$ Correlation is significant at the 0.01 level (2-tailed).

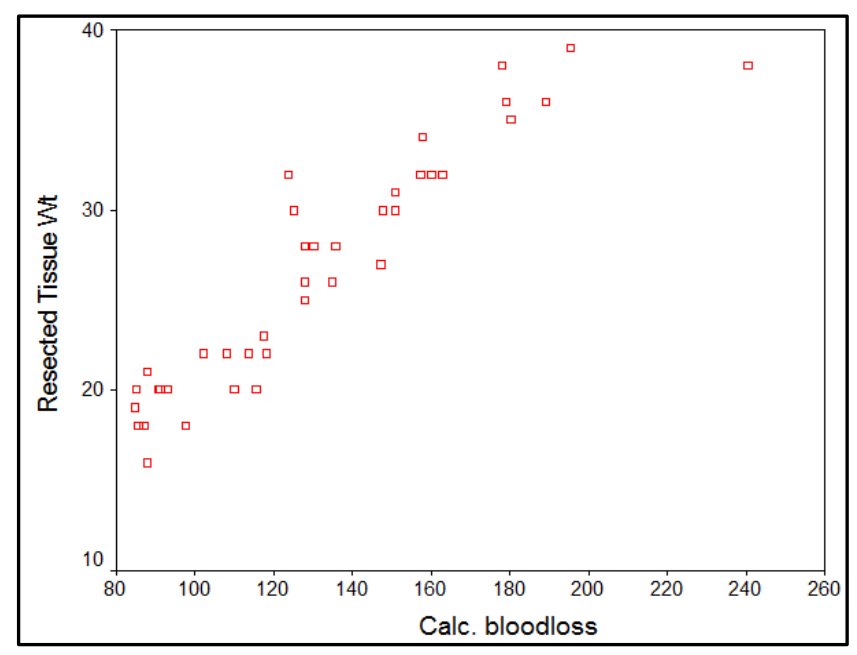

Graph 5

Group Statistics

\begin{tabular}{|c|c|c|c|c|c|}
\hline & $\begin{array}{c}\text { Urinary } \\
\text { Retention }\end{array}$ & $\mathbf{N}$ & Mean & $\begin{array}{c}\text { Std. } \\
\text { Deviation }\end{array}$ & $\begin{array}{c}\text { Std. } \\
\text { Error } \\
\text { Mean }\end{array}$ \\
\hline $\begin{array}{c}\text { Calc. } \\
\text { Blood } \\
\text { Loss }\end{array}$ & No & 17 & 116.759 & 29.5810 & 7.1744 \\
\cline { 2 - 6 } & Yes & 23 & 139.422 & 39.7409 & 8.2866 \\
\hline \multicolumn{5}{|c|}{$\begin{array}{r}\text { Table 8. Correlation between Urinary } \\
\text { Retention and Blood Loss }\end{array}$} \\
\hline
\end{tabular}

T - Test

\begin{tabular}{|c|c|c|c|c|c|c|}
\hline \multicolumn{7}{|c|}{ T-Test for Equality of Means } \\
\hline \multirow[t]{2}{*}{+} & \multirow[t]{2}{*}{ 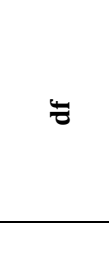 } & \multirow[t]{2}{*}{ 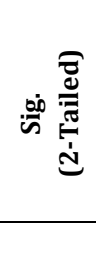 } & \multirow[t]{2}{*}{ 芯导 } & \multirow[t]{2}{*}{ 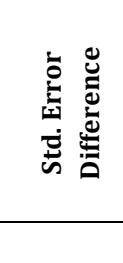 } & \multicolumn{2}{|c|}{ 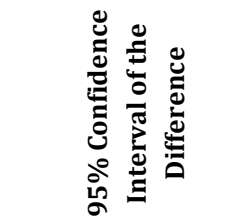 } \\
\hline & & & & & Lower & Upper \\
\hline $\begin{array}{c}- \\
1.978\end{array}$ & 38 & .055 & $\begin{array}{c}- \\
22.663\end{array}$ & 11.4556 & $\begin{array}{c}- \\
45.8536\end{array}$ & .5278 \\
\hline $\begin{array}{c}- \\
2.068\end{array}$ & 37.991 & .046 & $\begin{array}{c}- \\
22.663\end{array}$ & 10.9608 & $\begin{array}{c}- \\
44.8521\end{array}$ & -.4737 \\
\hline & & le 9. It & depende & t Sample & Test & \\
\hline
\end{tabular}




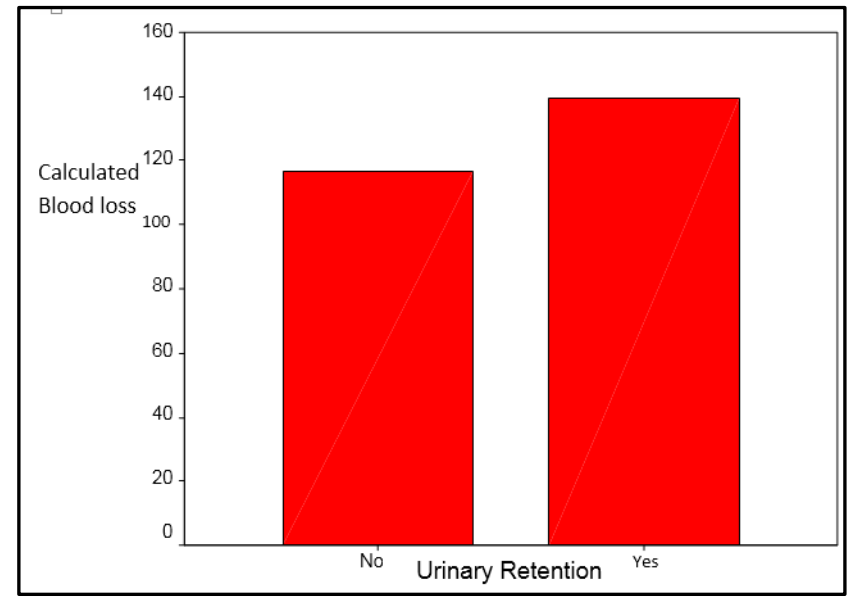

Graph 6

\begin{tabular}{|c|c|c|c|c|c|}
\hline \multicolumn{6}{|c|}{ Group Statistics } \\
\hline & Histology & $\mathbf{N}$ & Mean & $\begin{array}{c}\text { Std. } \\
\text { Deviation }\end{array}$ & $\begin{array}{c}\text { Std. } \\
\text { Error } \\
\text { Mean }\end{array}$ \\
\hline \multirow{2}{*}{$\begin{array}{l}\text { Calc. } \\
\text { Blood } \\
\text { Loss }\end{array}$} & Benign & 38 & 128.758 & 37.8305 & 6.1369 \\
\hline & Malignant & 2 & 149.400 & 2.1213 & 1.5000 \\
\hline \multicolumn{6}{|c|}{$\begin{array}{c}\text { Table 10: Correlations between Histology and } \\
\text { Calculated Blood Loss }\end{array}$} \\
\hline
\end{tabular}

$\mathrm{T}$ - Test

\begin{tabular}{|c|c|c|c|c|c|c|}
\hline \multicolumn{7}{|c|}{ T - Test for Equality of Means } \\
\hline \multirow[t]{2}{*}{-} & \multirow[t]{2}{*}{$\Xi$} & \multirow[t]{2}{*}{ क人 } & \multirow[t]{2}{*}{ 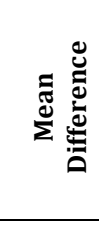 } & \multirow[t]{2}{*}{ 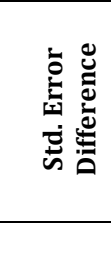 } & \multicolumn{2}{|c|}{ 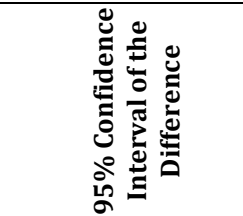 } \\
\hline & & & & & Lower & Upper \\
\hline-.762 & 38 & .451 & $\begin{array}{c}- \\
20.642\end{array}$ & 27.0828 & $\begin{array}{c}- \\
75.4683\end{array}$ & 34.1841 \\
\hline $\begin{array}{c}- \\
3.267\end{array}$ & 36.706 & .002 & $\begin{array}{c}- \\
20.642\end{array}$ & 6.3176 & $\begin{array}{c}- \\
33.4462\end{array}$ & -7.8380 \\
\hline
\end{tabular}

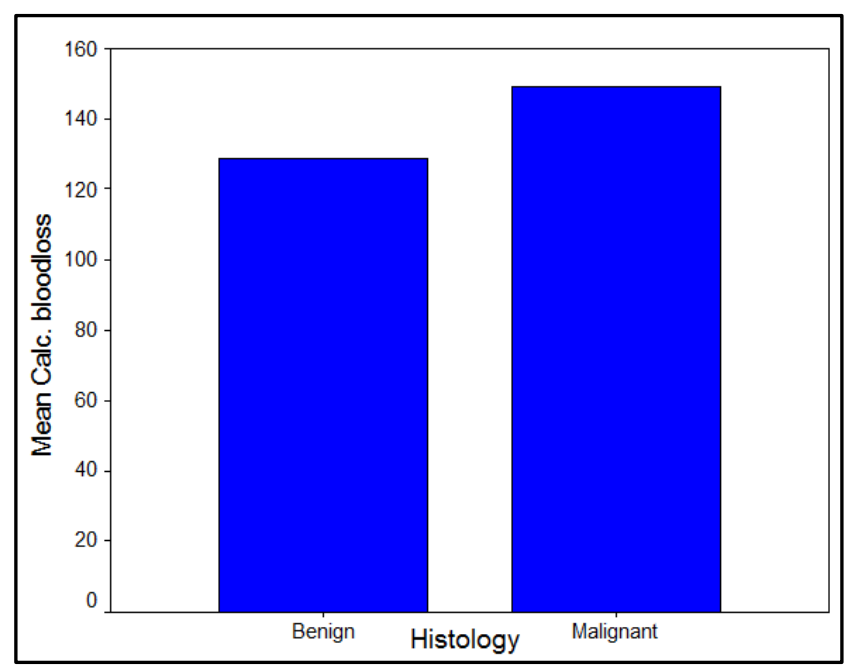

Graph 7

\section{Histology}

Malignant no. of patients: only 2 cases.

Mean blood loss $149.4 \mathrm{~mL}$.
Benign no. of patients: 38 cases.

Mean blood loss $128.752 \mathrm{~mL}$.

$P$ value is 0.451 . Not statistically significant.

\section{Type of Presentation}

Acute retention no. of patients: 23. Mean blood loss 139.422 $\mathrm{mL}$.

No retention no. of patients: 17. Mean blood loss $116.750 \mathrm{~mL}$. $P$ value is 0.055 . Not statistically significant correlation.

Our study of calculating blood loss is based on the calculation

Blood loss in $\mathrm{ml}=\frac{\text { Hb content of the irrigants }(\mathrm{gm} / \mathrm{L}) \mathrm{X} \text { Volume }(\mathrm{L}) \times 1000}{\text { Blood Hemoglobin }(\mathrm{gm} / \mathrm{dl}) \times 5.2}$

Shrestha BM et $\mathrm{al}^{6}$ studies showed mean blood loss was $95 \mathrm{~mL}$ (5 - 936).

In our study, the mean blood loss is $129.8 \mathrm{~mL}(84.6$ 240.8). This value is about $34.8 \mathrm{~mL}$ more.

Jan Eken Gren and Robert G. Hahn, ${ }^{7}$ mean blood loss is $300 \mathrm{~mL}(10$ - 3825).

In our study, mean blood loss is $129.8 \mathrm{~mL}$ (84.6 - 240.8). The blood loss is $170.2 \mathrm{~mL}$ less than the study.

Aiden M O'Donnell et al, ${ }^{8}$ Anaesthesia for TURP study mean blood loss is $500 \mathrm{~mL}$.

In our study, mean blood loss is $129.8 \mathrm{~mL}$. The blood loss is $370 \mathrm{~mL}$ less than the above study.

Tim Fagerstrom Stockholm Sweden. Study mean blood loss is $855 \mathrm{~mL}$ in our study, mean blood loss is $129.8 \mathrm{~mL}$. The blood loss is $725.2 \mathrm{~mL}$ less.

In our study, only one patient was transfused a single unit of blood post-operatively. The mean blood loss is lesser when compared to other 3 studies and it is more when compared with one study.

\section{Age of the Patients}

Age was not a significant influencing factor in almost all studies. In our study, mean age of the patient is 68.93 (5685). $P$ value is 0.185 , statistically not significant.

\section{Pre-Operative Haemoglobin and Calculated Blood Loss}

Tim Fagerstrom Stockholm Sweden. Study mean preoperative $\mathrm{Hb}$ was 14.18 gms\%. No statistically significant correlation.

No significant influence was noted in other studies.

In our study, the mean preoperative haemoglobin is 11.29 gms\% (9.4 - 13.6 gms $\%)$.

$P$ value is 0.015 , statistically not significant which correlates well with other studies.

\section{Gland Size and Calculated Blood Loss}

Shrestha B. M. et al ${ }^{6}$ study, the gland size had no significant influence on blood loss.

M. M. Kirollos and N. Campbell, ${ }^{9}$ Jan Eken Gren, Robert G. Hahn ${ }^{10}$ and Gordon ${ }^{11}$ had not taken the factor for the study.

Tim Fagerstrom Stockholm Sweden, study mean prostate volume was 58.2 gms. No significant correlations.

In our study, the mean gland size is 53.8 gms (30 - 82). $\mathrm{P}$ value is 0.000 , which is statistically significant. In one study, the gland size had no influence on blood loss. In our study, the gland size has significant influence on blood loss. 


\section{Resection Time}

Shrestha B. M. study ${ }^{6}$ - There was no statistically significant correlation between resection time and calculated blood loss. Jan Eken Gren and Robert G. Hahn, ${ }^{7}$ there was a strong correlation between operating time and blood loss ( $\mathrm{r}=0.72$; $\mathrm{P}<0.0001$ ).

Martin Marszalek et al, Vienna, Austria study resection time has significant correlation with blood loss.

Aiden $0^{\prime}$ Donnell et $\mathrm{al}^{8}$ study, resection time $>1 \mathrm{hr}$. had significant correlation with blood loss.

Tim Fagerstrom Stockholm Sweden, study mean resection time was 60 mins. had significant correlation.

In our study, the mean resection time is 52.38 (35 - 70).

$P$ value is 0.007 , which has statistically significant correlation with blood loss.

Four studies had statistically significant correlation with blood loss and one study had no significant correlation. Similar to other studies, our study has significant correlation between blood loss and resection time.

\section{Resected Tissue Weight and Blood Loss}

M. M. Kirollos and N. Campbell ${ }^{9}$ study mean resected wt. was 34.2 gms.

$\mathrm{P}$ value was $<0.001$, most important factor influencing blood loss.

Jan Eken Gren and Robert G. Hahn, ${ }^{7}$ study mean weight of resected tissue was 16.7 ( 2 - 171); $p<0.0001$ shows strong linear correlation.

Shrestha B. M. study aide - Mean resected tissue weight was 20.6 (3 - 60). There was no statistically significant correlation.

Tim Fagerstrom Stockholm, Sweden, study mean resected tissue wt was 26 gms.

$\mathrm{P}$ value was $<0.001$, statistically significant correlation exists.

Aiden 0'Donnell et $\mathrm{al}^{8}$ study, resected tissue weight had statistically significant correlation between resected tissue weight and calculated blood loss.

In our study, mean resected tissue wt is 26.30 gms. $P$ value is 0.000 , which has statistically significant influential factor with blood loss.

In four studies significant correlation was present and in one study no correlation was seen. Our study is comparable with other studies, has statistically significant linear correlation between the resected tissue weight with blood loss. The blood loss increases as the resected tissue weight increases.

\section{Histology of Resected Tissue}

M. M. Kirollos and N. Campbell ${ }^{9}$ study, no difference in blood loss between patients with benign and malignant prostates.

Abram et al,12 greater blood loss and longer resection time in benign prostatic tissue.

Jan Eken Gren and Robert G. Hahn ${ }^{7}$ study, blood loss was lesser in malignant tissue $(215 \mathrm{~mL})$ than benign $(350 \mathrm{~mL}) . \mathrm{P}$ value was $<0.007$.

The degree of differentiation of carcinoma (high, moderate or low) had no bearing on the blood loss.

Shrestha B. M. study, ${ }^{6}$ the blood loss did not show any correlation with histology.

Aiden $0^{\prime}$ Donnell et al ${ }^{8}$ study shows no relationship of histology with blood loss.
In our study, benign tissue no. of patients 38. Mean blood loss $128.75 \mathrm{~mL}$.

Malignant tissue no. of patients 2. Mean blood loss 149.4 $\mathrm{mL}$.

$P$ value 0.002 , which is not statistically significant.

In 3 studies, no significant influence in blood loss between benign and malignant histology. In one study, more blood loss in malignant. In one study, less blood loss in malignant tissue. In our study, the no. of patients with malignancy is only two, so comparison with other studies is not valuable.

\section{Type of Presentation (Acute Urinary Retention or Not)}

M. M. Kirollos and N. Campbell ${ }^{9}$ study, there was no difference in blood loss between acute retention patients and no retention patients, possibly because of antibiotics used in catheterised patients.

Shrestha B. M6 studies, no correlation between blood loss and patients presented with acute urinary retention.

Tim Fagerstrom, Stockholm, Sweden study, no correlations between blood loss and acute retention patients.

Aiden O'Donnell et al study, ${ }^{8}$ increased blood loss in patients with catheters for acute retention.

In our study, the patients with acute retention no. of patients 23.

Mean blood loss, $139.422 \mathrm{~mL}$. P value 0.046 .

Without acute retention no. of patients 17. Mean blood loss $116.759 \mathrm{~mL}$.

$P$ value 0.005 . Both are statistically not significant.

In three studies, no correlation between blood loss and type of presentation. In one study, blood loss increases with patients on catheter for acute retention. Explanation for this increase is infection in most catheterised patients causes more bleeding during prostatic resection.

Our study, although slight increase in blood loss in acute retention patients, no statistically significant increased blood loss in acute retention patients, possibly because antibiotic prophylaxis was used routinely for all catheterised patients.

\section{REFERENCES}

1. Thomson Walker JW. On the surgical anatomy of the prostate. J Anat Physiol 1906;40(Pt3):189-209.

2. McNeal JE, Mark AS, Marotti M, et al. MR imaging of the prostate gland: normal anatomy. American Journal of Roentgenology 1987;148(1):51-8.

3. Cho SY, Lee KS, Kim JH, et al. Effect of combined systematized behavioral modification education program with desmopressin in patients with nocturia: a prospective, multicenter, randomized, and parallel study. Int Neurourol J 2014;18(4):213-20.

4. Shrivastava A, Gupta VB. Various treatment options for benign prostatic hyperplasia: a current update. J Midlife Health 2012;3(1):10-9.

5. Rassweiler J, Teber D, Kuntz R, et al. Complications of transurethral resection of the prostate-incidence, management and prevention. Eur Urol 2006;50(5):96980.

6. Shrestha B, Baidhya JL. Morbidity and early outcome of transurethral resection of prostate: a prospective singleinstitute evaluation of 100 patients. Kathmandu Univ Med J (KUMJ) 2010;8(30):203-7. 
7. Ekengren J, Hahn RG. Blood loss during transurethral resection of the prostate as measured by the HemoCue photometer. Scand J Urol Nephrol 1993;27(4):501-7.

8. O'Donnell AM. Anesthesia for transurethral resection of the prostate. Contin Educ Anaesth Crit Care Pain 2009;9(3):92-6.

9. Kirollos MM, Campbell N. Factors influencing blood loss in transurethral resection of the prostate (TURP): auditing TURP. Br J Urol 1997;80(1):111-5.
10. Sandfeldt L, Bailey DM, Hahn RG. Blood loss during transurethral resection of the prostate after 3 months of treatment with finasteride. Urology 2001;58(6):972-6.

11. Desmond JW, Gordon RA. Bleeding during transurethral prostatic surgery. Can Anaesth Soc J 1969;16(3):217-24.

12. Abrams PH, Shah PJ, Bryning K, et al. Blood loss during transurethral resection of the prostate. Anesthesia 1982;37(1):71-3. 